\title{
Overexpression of synuclein- $\gamma$ predicts lack of benefit from radiotherapy for breast cancer patients
}

\author{
Li Min ${ }^{1,2+} \mathbb{D}$, Cheng Zhang ${ }^{1 \dagger}$, Ruolan Ma ${ }^{3}$, Xiaofan $\mathrm{Li}^{4}$, Hua Yuan ${ }^{5}$, Yihao Li ${ }^{2,6}$, Ruxuan Chen , Caiyun Liu',
} Jianping Guo', Like Qu ${ }^{1 *}$ and Chengchao Shou ${ }^{1 *}$

\begin{abstract}
Background: Although radiotherapy following mastectomy was demonstrated to reduce the recurring risk and improve the prognosis of patients with breast cancer, it is also notorious for comprehensive side effects, hence only a selected group of patients can benefit. Therefore, the screening of molecular markers capable of predicting the efficacy of radiotherapy is essential.

Methods: We have established a cohort of 454 breast cancer cases and selected 238 patients with indications for postoperative radiotherapy. Synuclein- $\gamma$ (SNCG) protein levels were assessed by immunohistochemistry, and SNCG status was retrospectively correlated with clinical features and survival in patients treated or not treated with radiotherapy. Gene Set Enrichment Analysis (GSEA) and survival analysis for online datasets were also performed for further validation.

Results: Among patients that received radiotherapy (82/238), those demonstrating positive SNCG expression had a 55. 0 month shorter median overall survival (OS) in comparison to those demonstrating negative SNCG expression (78.4 vs. 133.4 months, log rank $\left.X^{2}=16.13 ; p<0.001\right)$. Among the patients that received no radiotherapy $(156 / 238)$, SNCG status was not correlated with OS ( $\log$ rank $x^{2}=2.40 ; p=0.121$ ). A COX proportional hazard analysis confirmed SNCG as an independent predictor of OS, only for patients who have received radiotherapy. Similar results were also obtained for distant metastasis-free survival (DMFS). A GSEA analysis indicated that SNCG was strongly associated with genes related to a radiation stress response. A survival analysis was performed with online databases consisting of breast cancer, lung cancer, and glioblastoma and further confirmed SNCG's significance in predicting the survival of patients that have received radiotherapy.
\end{abstract}

Conclusion: A positive SNCG may serve as a potential marker to identify breast cancer patients who are less likely to benefit from radiotherapy and may also be extended to other types of cancer. However, the role of SNCG in radiotherapy response still needs to be further validated in randomized controlled trials prior to being exploited in clinical practice.

Keywords: Synuclein- $\gamma$, Radiotherapy, Prognosis, Breast cancer

Abbreviations: $\mathrm{Cl}$, Confidence interval; DAB, Diaminobenzidine; DMFS, Distant metastasis-free survival; ER, Estrogen receptor; FDR, False discovery rate; GSEA, Gene set enrichment analysis; HR, Hazard ratios; IHC, Immunohistochemistry; MSigDB, Molecular signatures database; NES, Normalized enrichment score; OS, Overall survival; ROS, Reactive oxygen species; SNCG, Synuclein- $\gamma$

\footnotetext{
* Correspondence: qulike@bjcancer.org; scc@bjcancer.org

†Equal contributors

${ }^{1}$ Department of Biochemistry and Molecular Biology, Key Laboratory of

Carcinogenesis and Translational Research (Ministry of Education), Peking

University Cancer Hospital \& Institute, Beijing 100142, China

Full list of author information is available at the end of the article
}

\section{Biomed Central}

(c) 2016 The Author(s). Open Access This article is distributed under the terms of the Creative Commons Attribution 4.0 International License (http://creativecommons.org/licenses/by/4.0/), which permits unrestricted use, distribution, and reproduction in any medium, provided you give appropriate credit to the original author(s) and the source, provide a link to the Creative Commons license, and indicate if changes were made. The Creative Commons Public Domain Dedication waiver (http://creativecommons.org/publicdomain/zero/1.0/) applies to the data made available in this article, unless otherwise stated. 


\section{Background}

Breast cancer is the most frequently diagnosed cancer among females worldwide [1]. In more developed countries like the U.S., breast cancer death rates have slowly decreased by $1.4 \%$ per year [2, 3]; however, in less developed areas, both of the incidence rate and mortality rate of breast cancer are still raising [1]. In 2013, breast cancer accounted for $25 \%$ of total cancer cases and $15 \%$ of cancer-related deaths worldwide [1]. For decades, surgical removal of the primary tumor has been the major therapeutic option $[4,5]$, and the addition of adjuvant radiotherapy based on a risk of recurrence and metastasis has been found to significantly improve the overall prognosis. Currently, adjuvant radiotherapy after mastectomy has been widely accepted as the gold standard of care for patients with tumors $>5 \mathrm{~cm}$ in size, 4 or more positive lymph nodes, or positive margins [6]. However, radiotherapy is also associated with potential long-term side effects and radiation oncologists have to be highly selective of patients and administer radiation treatments with extreme caution $[6,7]$. Despite such precautions, not every patient subjected to radiotherapy can particularly benefit from it. Thus, biomarkers capable of predicting radiotherapeutic efficacy would largely strengthen current clinical options by providing instructions for appropriate risk evaluation and treatment plan selection.

Synuclein- $\gamma$ (SNCG) was first identified as breast cancer-specific gene 1 (BCSG1), and was isolated from cDNA libraries of breast carcinoma in the 1990s [8, 9]. SNCG is highly expressed in advanced and metastatic breast tumors but not in normal breast epithelium tissues. In breast cancer cells, SNCG protein impairs cell cycle checkpoints [10, 11], confers chemoresistance [12, 13], and enhances metastasis in nude mice [14]. Although the detailed mechanism is not fully understood, SNCG's role in the oncogenesis-related Akt and mTOR pathways [15] and the neural development-related PPARY pathway [16] are noteworthy and worth further investigation. The poor overall SNCG-related prognosis in breast cancer has been reported by two independent studies $[17,18]$. Moreover, SNCG was overexpressed in other cancerous tissues and this overexpression was a prediction of poor prognosis in several types of cancer [17-22]. Nevertheless, the relationship between SNCG expression and radiotherapeutic efficacy remains to be elucidated.

The aim of this study is to explore the impact of SNCG expression on the prognosis as well as multiple clinical manifestations of breast cancer patients treated with radiotherapy. Surgically resected specimens from breast cancer patients as well as expression profiling datasets from online repositories were simultaneously analyzed. SNCG expression and its relationship with pathological parameters were investigated on both protein and transcript levels, and high SNCG expression were suggested to be an indication of fewer radiotherapeutic benefits. Furthermore, our finding was also validated by analysis performed in two online datasets of different cancer types with radiotherapy information. In conclusion, this study has revealed the prospective value of SNCG expression in predicting whether breast cancer patients could benefit from radiotherapy, and could further potentially be used as a significant parameter for cancer adjuvant treatment.

\section{Methods \\ Patient selection}

A cohort of 454 invasive breast cancer patients that received radical or modified radical mastectomy between the years of 1996 and 2002 in the Breast Center at the Peking University Cancer Hospital \& Institute. The project was approved and supervised by the research ethics committee of Peking University Cancer Hospital \& Institute. Written informed consents were obtained from all participants. Patients with indications for postoperative radiotherapy were recruited: patients with T3/4 tumors (i.e. tumor size $>5 \mathrm{~cm}$ in size, or positive margins), patients with four or more positive lymph nodes, T1/2 patients with one to three positive nodes and other risk factors of recurrence (i.e. $\leq 40$ years old, hormone receptor-negative, HER2 positive, incomplete lymph node dissection or more than $20 \%$ positive nodes). The presence of ER and PR was evaluated using the charcoal-dextran method. ER and PR values of more than $10 \mathrm{fmol} / \mathrm{mg}$ were considered positive. Status of HER2 was assessed by IHC with a rabbit polyclonal antibody (DAKO A0485; 1:250 dilution), and scored by the Diagnostic Pathological Department, Peking University Cancer Hospital. Eight fields were randomly selected in each slide and slides were counted under a Nikon microscope at 200x amplification [17]. Among the 454 breast cancer patient cohort, 238 of the cases with indications for postoperative radiotherapy were selected while only 82 of them had been treated with radiotherapy. No patients involved in this study have received neoadjuvant chemotherapy.

\section{Radiotherapy treatment}

Overall, there were 238 patients with indications for postoperative radiotherapy that were selected. The selected patients were aged from 25 to 81 years (median 52 years). 82 of them were typically treated with standard radiotherapy in 25 fractions (50 Gy at 2 Gy per fraction, 5 fractions per week), and ensured that the radiotherapy dose was actually delivered to the CTV (clinical target volume) with $6 \mathrm{MV}$ photons or electron beam. The remaining 156 patients had not been subjected to radiotherapy. 


\section{Clinical samples handling}

Surgically resected tissue specimens were used in this study. Formalin fixed, paraffin-embedded breast cancer tissue specimens from the above 238 patients were obtained from the Breast Center at Peking University Cancer Hospital \& Institute. The study was approved and supervised by the Medical Ethics Committee of Peking University Cancer Hospital \& Institute and each patient had given formal consent. All specimens were taken before the onset of chemotherapy or hormonal treatment. The total period of follow-up was 60-192 months with a median period of 127 months.

\section{Immunohistochemical staining}

Specimens were cut into $5 \mu \mathrm{m}$ sections. After baking at $60{ }^{\circ} \mathrm{C}$ overnight, sections were dewaxed and rehydrated through xylene and alcohol series. Antigen retrieval was performed via microwave cooking in ethylene diamine tetra acetic acid ( $\mathrm{pH}$ 8.0, Zymed) for $20 \mathrm{~min}$. Endogenous peroxidase activity was blocked by incubation in $3 \%$ hydrogen peroxide for $10 \mathrm{~min}$ at room temperature. Non-specific binding was blocked with $10 \%$ goat serum. Then slides were subjected to overnight incubation at $4{ }^{\circ} \mathrm{C}$ with anti-SNCG monoclonal antibody generated in our laboratory [17]. After incubation with a biotin-conjugated secondary anti-mouse antibody for $30 \mathrm{~min}$ and 3 washes with phosphate-buffered saline with $0.1 \%$ Tween-20, slides were treated with diaminobenzidine (DAB) working solution at room temperature for 3-10 $\mathrm{min}$, and then washed in distilled water and counterstained with hematoxylin. The negative control was prepared by replacing the SNCG antibody with non-immune IgG in a randomly selected breast cancer tissue slide, and the positive control was prepared with SNCG antibody in a known SNCG positive breast cancer tissue slide which had been proved in a previous study [17].

\section{IHC grading system}

All of the samples were independently inspected under a light microscope (APPLIED IMAGING at 200x) by two experienced pathologists. Both the percentage of positive cells and the intensity of staining in 10 randomly chosen microscopic fields were evaluated. According to our previous publications, the grading system was based on a 4value classification scale as follows: the area of staining was graded as $<10 \%(0)$ or $>10 \%$ (1) of all cancer cells stained within the section; intensity of staining was graded as none (0), weak (1), moderate (2) or strong (3). The final grade was obtained by adding area grade and intensity grade together, and final grade $\geq 3$ was defined as positive [20, 23].

\section{Validating analysis}

EBI ArrayExpress dataset E-TABM-158 and NCBI GEO dataset GSE1456 are two online breast cancer datasets with radiotherapy information [24, 25]. Both of the two datasets with their supplementary clinical information were downloaded and used for validating analysis. A pearson correlation analysis was performed to assess the gene-gene expression correlation. A hierarchical clustering was used to distinguish different subgroups according to expression level of given genes. Gene Set Enrichment Analysis (GSEA) was performed to evaluate correlation between SNCG expression and two radiation stress response gene sets [26, 27]. Lung cancer dataset CaArray and glioblastoma dataset GSE13041 with radiotherapy information were also downloaded and used for validating analysis [28-30].

\section{Statistical analysis}

Since the populations of stage II patients in the radiotherapy subgroup were too small to perform a separate multivariate analysis, we combined the samples in stage II and III to make it sufficient for statistics. All statistical analyses were performed using the $\mathrm{R}$ 3.1.2 software (www.r-project.org). Correlations that were made between the SNCG expression and clinicopathologic characteristics were tested by the Pearson $\chi^{2}$ test. The Kaplan-Meier curve was used to evaluate overall survival (OS) and distant metastasis-free survival (DMFS) rates, and differences were tested by log-rank test. The COX proportional hazard model was used for multivariate analysis. Hazard ratios (HR) and $95 \%$ confidence interval (CI) were calculated. All statistical analyses were 2 -sided, and a $p$ value less than 0.05 were considered statistically significant. For false discovery rate (FDR) analysis, a cutoff of 0.25 was selected according to GSEA's suggestion [26].

\section{Results}

\section{Association of SNCG expression and clinicopathologic features}

IHC staining of SNCG was performed for all samples. According to our grading criteria, 139 samples among 238 were defined as SNCG negative while another 99 were defined as SNCG positive (total positive rate= $41.6 \%)$. Representative images of SNCG staining in breast cancer tissues with examples of scoring were shown in Fig. 1. Positive rates of SNCG were $41.5 \%$ $(34 / 82)$ in patients that received radiotherapy and $41.7 \%(65 / 156)$ in those that did not receive radiotherapy, and there was no significant difference $\left(\chi^{2}=\right.$ $0.001, p=0.976)$. For patients treated with radiotherapy or not, there were no significant associations between SNCG expression and Age ( $p=0.767,0.665)$, Tumor size $(p=0.145,0.142)$, Metastasis lymph node $(p=0.117,0.332)$, TNM stage $(p=0.428,0.957)$, ER status $(p=0.304,0.998), \mathrm{PR}$ status $(p=0.171,0.904)$, or HER2 status $(p=0.351,0.646)$, and all of the 

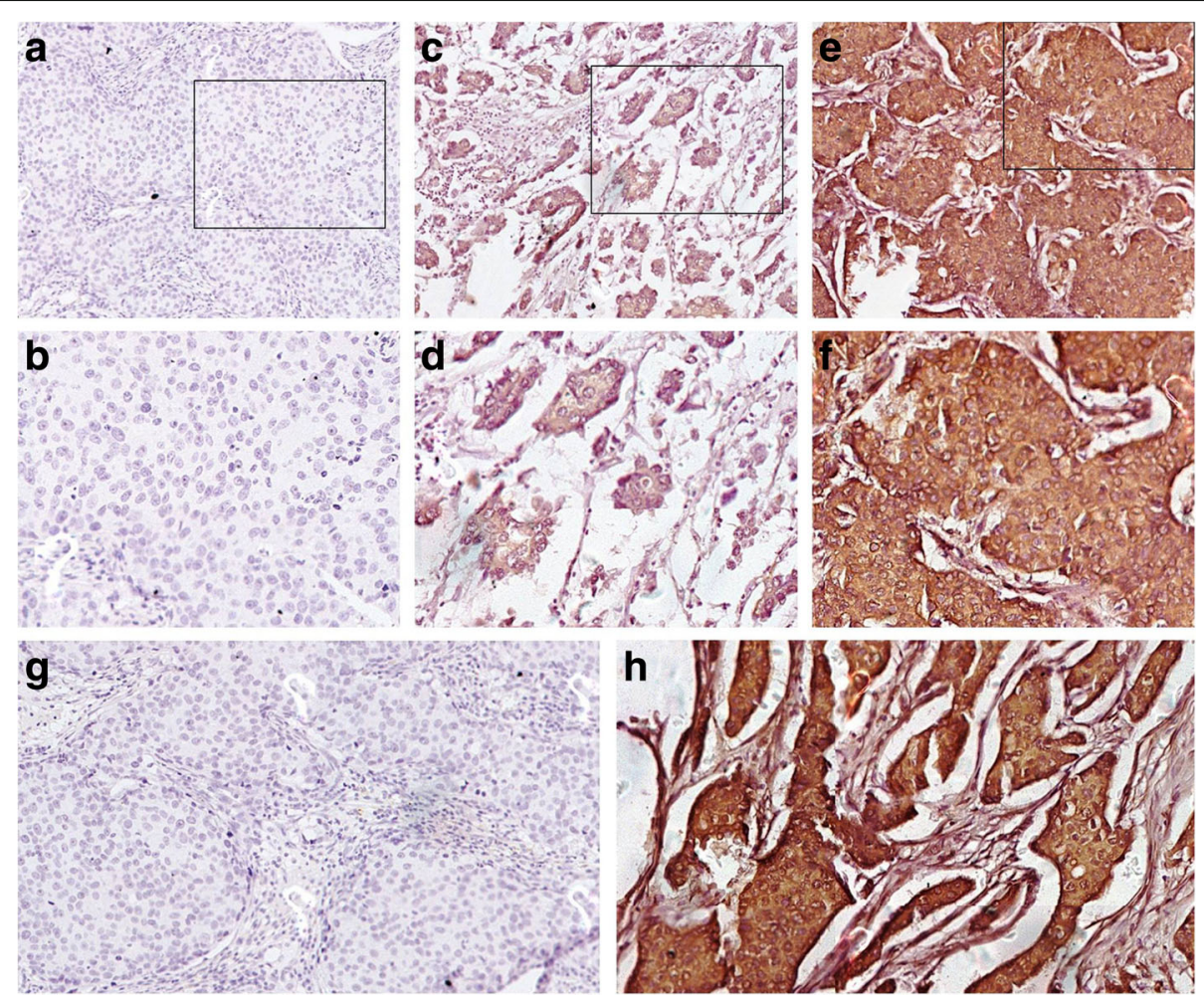

Fig. 1 Representative immunohistochemical staining for SNCG expression in breast cancer tissues. a $100 \times$ and $\mathbf{b} 200 \times$ staining of negative sample 1 (area grade 0, intensity grade 0); c $100 \times$ and $\mathbf{d} 200 \times$ staining of negative sample 2 (area grade 1, intensity grade 1 ); e $100 \times$ and $\mathbf{f}$ $200 \times$ staining of positive sample (area grade 1, intensity grade 3); $\mathbf{g}$ Staining of negative control (100x); $\mathbf{h}$ Staining of positive control (100x)

clinicopathologic features in both subgroups were equally distributed (Table 1).

\section{Relationship between SNCG expression and radiotherapy stratified survival}

Positive SNCG was correlated with decreased OS (median OS: 108.3 vs. 144.6 months; log rank $X^{2}=$ 13.45; $p<0.001$; Fig. 2a) and DMFS (median DMFS: 81.2 vs. 127.7 months; $\log \operatorname{rank} \chi^{2}=17.83 ; p<0.001$; Fig. 2d) in breast cancer patients, regardless of the utilization or non-utilization of radiotherapy.

Among patients that received radiotherapy, those with positive SNCG expression had a 55.0 months shorter median OS than those with negative SNCG expression (median OS: 78.4 vs. 133.4 months; $\log$ rank $\chi^{2}=16.13$; $p<0.001$; Fig. 2b). However, among patients that were not subjected to radiotherapy, there was no significant difference between OS of patients with positive SNCG expression and those with negative SNCG expression (median OS: 122.4 vs. 143.1 months; $\log$ rank $\chi^{2}=2.40$; $p=0.121$; Fig. 2c). Similar results were also obtained for DMFS (for patients received radiotherapy, median DMFS: 52.9 vs. 116.7 months, Fig. 2e; for patients did not receive radiotherapy, median DMFS: 95.1 vs. 126.7 months, Fig. 2f).

\section{Univariate and multivariate analysis for the radiotherapy stratified prognosis}

In univariate analysis, tumor size, lymph nodes metastasis, TNM stage, SNCG expression were statistically associated with $O S$ in patients that received radiotherapy, while lymph nodes metastasis, TNM stage, and HER2 status were prognostic factors of OS in patients that did not receive radiotherapy (Table 2 ).

Multivariate analyses using COX regression analysis identified TNM stage (Wald $\chi^{2}=10.31 ; p=0.001$ ) and SNCG (Wald $\chi^{2}=6.62 ; p=0.010$ ) expression, which were both independent predictors of OS in patients that received radiotherapy. However, in patients that did not receive radiotherapy, only TNM stage (Wald $\chi^{2}=7.32$; $p=0.007$ ) remained an independent prognostic factor (Table 3). Similar results were also obtained for DMFS (Additional file 1: Table S1 and S2). Taken together, SNCG expression affected the survival of breast cancer patients to a greater extent in patients that received radiotherapy.

\section{Association between SNCG expression and radiation stress response gene sets}

SMIRNOV_RESPONSE_TO_IR_2HR_DN gene set includes a series of genes that are down-regulated in 
Table 1 Association of SNCG expression with clinicopathological parameters in breast cancer patients were or were not treated with radiotherapy

\begin{tabular}{|c|c|c|c|c|c|c|c|c|}
\hline \multirow[t]{2}{*}{ Characteristics } & \multicolumn{4}{|c|}{ Radiotherapy } & \multicolumn{4}{|c|}{ No Radiotherapy } \\
\hline & SNCG- & SNCG+ & $x^{2}$ & $p$-value & SNCG- & SNCG+ & $x^{2}$ & $p$-value \\
\hline Age & & & 0.088 & 0.767 & & & 0.188 & 0.665 \\
\hline$<50$ years & 21 & 16 & & & 43 & 33 & & \\
\hline$\geq 50$ years & 27 & 18 & & & 48 & 32 & & \\
\hline Tumor size & & & 2.126 & 0.145 & & & 2.161 & 0.142 \\
\hline$<2 \mathrm{~cm}$ & 29 & 15 & & & 40 & 21 & & \\
\hline$\geq 2 \mathrm{~cm}$ & 19 & 19 & & & 51 & 44 & & \\
\hline Metastasis lymph node & & & $4.393^{\mathrm{a}}$ & 0.117 & & & 2.202 & 0.332 \\
\hline 0 & 19 & 7 & & & 34 & 17 & & \\
\hline $1-3$ & 6 & 3 & & & 31 & 27 & & \\
\hline$\geq 4$ & 23 & 24 & & & 26 & 21 & & \\
\hline TNM stage & & & 0.628 & 0.428 & & & $0.003^{a}$ & 0.957 \\
\hline 1 & 12 & 6 & & & 7 & 4 & & \\
\hline$\|\|$, & 36 & 28 & & & 84 & 61 & & \\
\hline$E R$ & & & 1.058 & 0.304 & & & 0.001 & 0.998 \\
\hline negative & 12 & 13 & & & 34 & 25 & & \\
\hline positive & 32 & 21 & & & 53 & 39 & & \\
\hline$P R$ & & & 1.874 & 0.171 & & & 0.014 & 0.904 \\
\hline negative & 23 & 23 & & & 44 & 33 & & \\
\hline positive & 21 & 11 & & & 43 & 31 & & \\
\hline HER2 & & & 0.871 & 0.351 & & & 0.211 & 0.646 \\
\hline negative & 28 & 21 & & & 50 & 41 & & \\
\hline positive & 7 & 9 & & & 14 & 14 & & \\
\hline
\end{tabular}

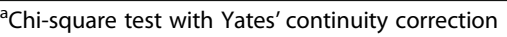
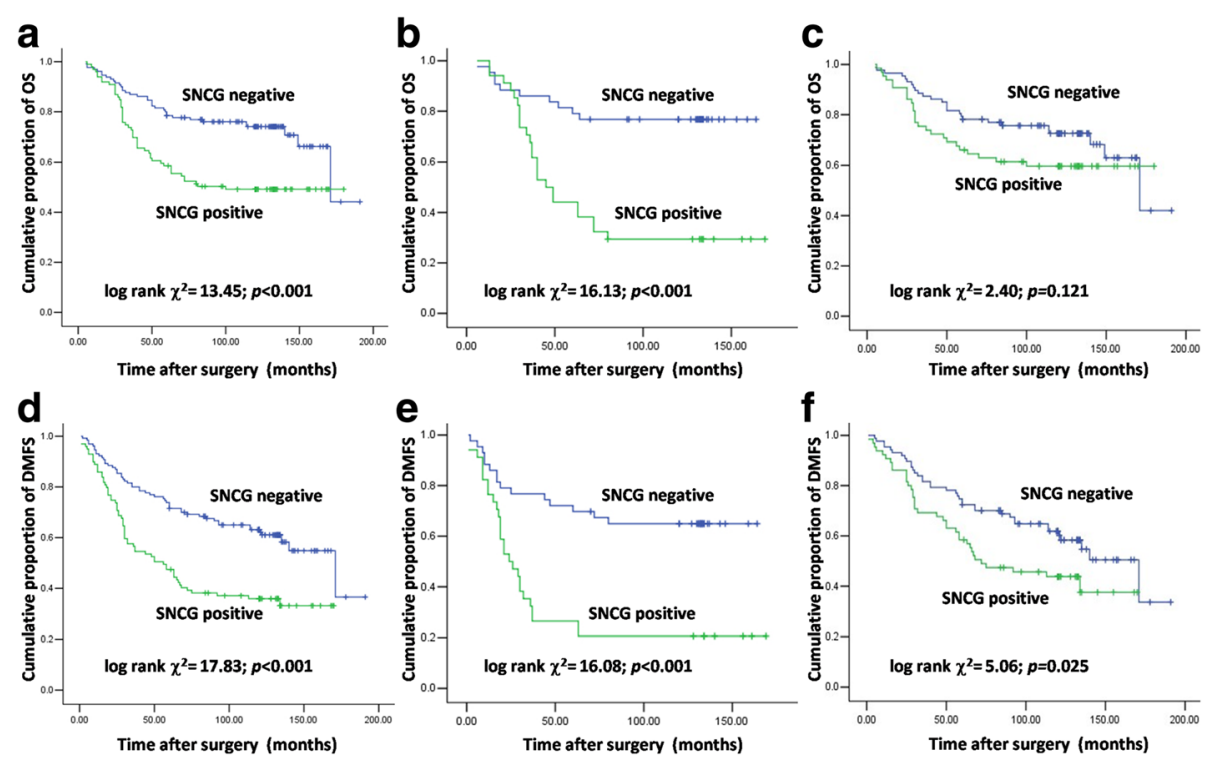

Fig. 2 Kaplan-Meier curve of OS/DMFS in breast cancer patients evaluated according to SNCG expression, stratified with radiotherapy reception. All patients $(\mathbf{a}, \mathbf{d})$; patients received radiotherapy $(\mathbf{b}, \mathbf{e})$; patients did not receive radiotherapy $(\mathbf{c}, \mathbf{f})$ 
Table 2 Prognostic factors of OS in univariate analysis of breast cancer patients were or were not treated with radiotherapy

\begin{tabular}{|c|c|c|c|c|}
\hline \multirow[t]{2}{*}{ Characteristics } & \multicolumn{2}{|l|}{ Radiotherapy } & \multicolumn{2}{|l|}{ No Radiotherapy } \\
\hline & $\mathrm{RR}(95 \% \mathrm{Cl})$ & $p$-value & $\mathrm{RR}(95 \% \mathrm{Cl})$ & $p$-value \\
\hline Age & & 0.429 & & 0.739 \\
\hline$\geq 50$ vs. $<50$ & $0.759(0.383,1.503)$ & & $1.098(0.635,1.898)$ & \\
\hline Tumor size & & 0.011 & & 0.730 \\
\hline$\geq 2 \mathrm{~cm}$ vs. $<2 \mathrm{~cm}$ & $2.432(1.203,4.918)$ & & $1.104(0.628,1.944)$ & \\
\hline Metastasis lymph node & & $<0.001$ & & 0.001 \\
\hline $1-3$ vs. 0 & $1.878(0.314,11.243)$ & & $0.935(0.433,2.017)$ & \\
\hline$\geq 4$ vs. 0 & $8.151(2.475,26.845)$ & & $2.763(1.415,5.396)$ & \\
\hline TNM stage & & $<0.001$ & & $<0.001$ \\
\hline II, III vs. I & $6.624(2.556,17.171)$ & & $2.906(1.681,5.025)$ & \\
\hline SNCG & & $<0.001$ & & 0.126 \\
\hline Positive vs. Negative & $4.058(1.929,8.537)$ & & $1.530(0.888,2.638)$ & \\
\hline ER & & 0.176 & & 0.183 \\
\hline Positive vs. Negative & $0.613(0.306,1.226)$ & & $1.530(0.818,2.861)$ & \\
\hline PR & & 0.485 & & 0.232 \\
\hline Positive vs. Negative & $0.782(0.391,1.561)$ & & $1.423(0.798,2.538)$ & \\
\hline HER2 & & 0.985 & & 0.018 \\
\hline Positive vs. Negative & $1.008(0.434,2.341)$ & & $2.304(1.152,4.606)$ & \\
\hline
\end{tabular}

RR Risk Ratio, Cl confidence interval

lymphocytes at $2 \mathrm{~h}$ after exposure to $10 \mathrm{~Gy}$ dose of ionizing radiation [31] and MAYBURD_RESPONSE_TO_L663536_DN gene set includes a series of genes down-regulated in cancer cells after treatment with L663536, a small molecular chemical found to enhance the effect of radiation in cancer cells [32]. These two gene sets located in the Molecular Signatures Database (MSigDB) were downloaded to perform GSEA analysis in online datasets E-TABM-158 and GSE1456.

In ArrayExpress dataset E-TABM-158, the Normalized Enrichment Score (NES) of MAYBURD_RESPONSE_TO_ L663536_DN gene set was $-1.571(p=0.049$, FDR $=0.238$, Fig. 3a), indicating SNCG expression is negatively correlated with genes in this gene set significantly. For SMIRNOV_RESPONSE_TO_IR_2HR_DN gene set, a NES of -1.532 was achieved $(p=0.036, \mathrm{FDR}=0.236$, Fig. 3b).

Table 3 Independent predictors of OS in multivariate analysis of breast cancer patients were or were not treated with radiotherapy

\begin{tabular}{llllll}
\hline Characteristics & Radiotherapy & & & No Radiotherapy & \\
\cline { 2 - 3 } \cline { 5 - 6 } \cline { 5 - 6 } & RR $(95 \% \mathrm{Cl})$ & $p$-value & & RR $(95 \% \mathrm{Cl})$ & $p$-value \\
\hline TNM stage & & 0.001 & & 0.007 \\
II, III vs. I & $4.960(1.866,13.183)$ & & & $2.548(1.294,5.019)$ & \\
SNCG & & 0.010 & & \\
$\begin{array}{c}\text { Positive vs. } \\
\text { Negative }\end{array}$ & $2.726(1.270,5.850)$ & & & \\
\hline
\end{tabular}

Similar results were also obtained in the NCBI GEO dataset GSE1456. NES of MAYBURD_RESPONSE_TO_L663536_DN was $-1.667(p=0.025$, FDR $=0.137$, Fig. 3c), while NES of MIRNOV_RESPONSE_TO_IR_2 HR_DN was $-1.569(p=0.028$, FDR $=0.196$, Fig. $3 \mathrm{~d})$.

\section{Relationship between SNCG expression and radiotherapy} stratified survival in validating dataset

A validating survival analysis was then conducted. Considering that the sample size of GSE1456 was too small to stratify, only the $E-T A B M-158$ dataset was used in the subsequent survival analyses. The E-TABM-158 dataset was stratified to the radiotherapy subgroup and the nonradiotherapy subgroup.

In the radiotherapy subgroup, patients with high SNCG levels had a worse DMFS values than those with low SNCG levels. A suggestive $p$-value was achieved, considering the small sample size (Fig. 3e, log-rank $\chi^{2}=2.45, p=$ $0.118)$. In the non-radiotherapy subgroup, patients with different SNCG levels had nearly the same survival outcome (Fig. 3f, log-rank $\chi^{2}=0.35, p=0.555$ ). Additionally, the percentage of censored data was too high to calculate the median DMFS in both subgroups.

To get a survival indicator of higher resolution, a set of SNCG correlated genes (the expression of those genes was correlated with SNCG in both E-TABM-158 and GSE1456 datasets) were recruited to construct a novel SNCG signature. In the radiotherapy subgroup, patients with different SNCG signatures showed a significant 

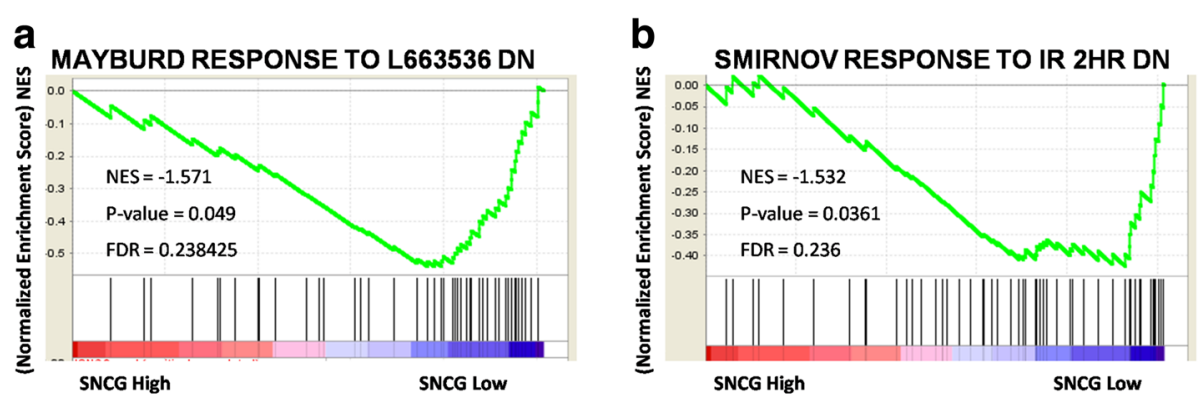

C

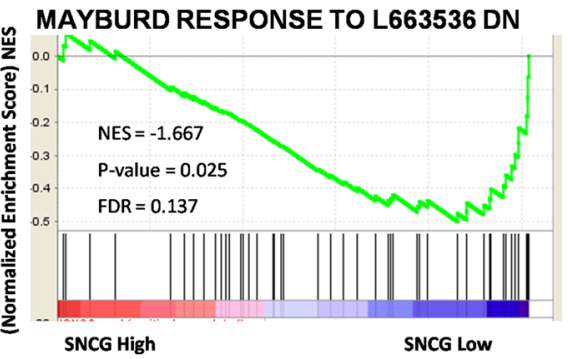

d
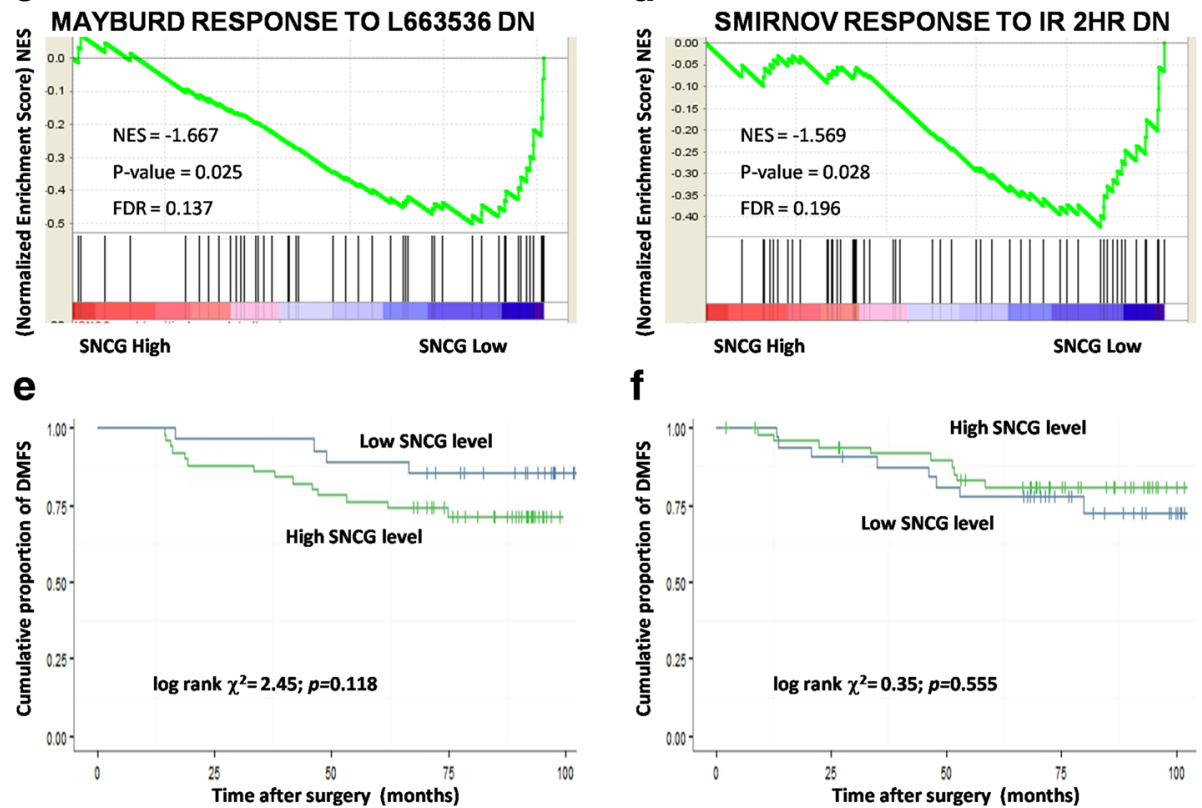

f

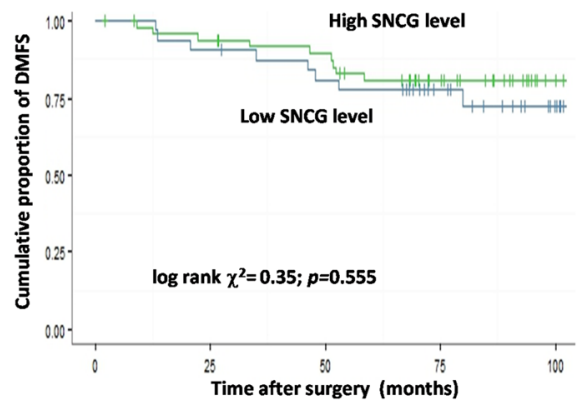

Fig. 3 SNCG expression is associated with radiation related GSEA gene sets and radiotherapy related survival. Normalized Enrichment Score (NES) of MAYBURD_RESPONSE_TO_L663536_DN gene set in breast cancer dataset E-TABM-158 (a) and GSE1456 (c); Normalized Enrichment Score (NES) of SMIRNOV_RESPONSE_TO_IR_2HR_DN gene set in breast cancer dataset E-TABM-158 (b) and GSE1456 (d); Kaplan-Meier curve of DMFS evaluated according to SNCG expression in patients received radiotherapy (e) and patients did not receive radiotherapy (f) in breast cancer dataset E-TABM-158

difference in DMFS (log-rank $\chi^{2}=3.87, p=0.049$, Fig. 4a, b), while in the non-radiotherapy subgroup, patients with different SNCG signatures had similar DMFS (log-rank $\chi^{2}=1.16, p=0.282$, Fig. 4c, d).

\section{Relationship between SNCG expression and radiotherapy stratified survival in other types of cancer}

Additionally, a relationship between SNCG expression and radiotherapy-related survival was further testified in other cancers. Pertaining to the lung cancer dataset CaArray, high SNCG levels indicated a significantly worse OS than low SNCG level in patients that received radiotherapy (median OS: 25.5 vs. 44.4 months; log rank $\chi^{2}=4.64 ; p=0.030$; Fig. 5a, c). Similar OS was observed regardless of the SNCG level in patients that did not receive radiotherapy (median OS: 75.7 vs. 79.5 months; log $\operatorname{rank} \chi^{2}=0.14 ; p=0.711$; Fig. 5b, d).

Regarding the glioblastoma dataset GSE13041, a high SNCG level indicated a marginally significantly worse
OS than low SNCG level in patients received radiotherapy (median OS: 6.0 vs. 11.5 months; $\log \operatorname{rank} \chi^{2}=3.61$; $p=0.050$; Fig. $5 \mathrm{e}, \mathrm{g})$. In patients that did not receive radiotherapy, a similar OS was observed regardless of the SNCG level (median OS: 14.5 vs. 14.0 months; log $\operatorname{rank} \chi^{2}=0.17 ; p=0.678$; Fig. 5f, h).

\section{Discussion}

Despite that the overexpression of SNCG has long been observed in cancers and high levels of SNCG expression has been validated to be associated with poorer OS and DMFS in multiple types of cancer, the suitability of SNCG expression as a biomarker for patient selection in radiotherapy remains largely unknown [17-21]. Kang et al. reported that radiotherapy induced SNCG expression in the MCF7 cell line, which may contribute to immune suppressive effects by inhibiting the differentiation and activation of dendritic cells. Yet the linkage between SNCG and radiotherapy remains to be elucidated [33]. 


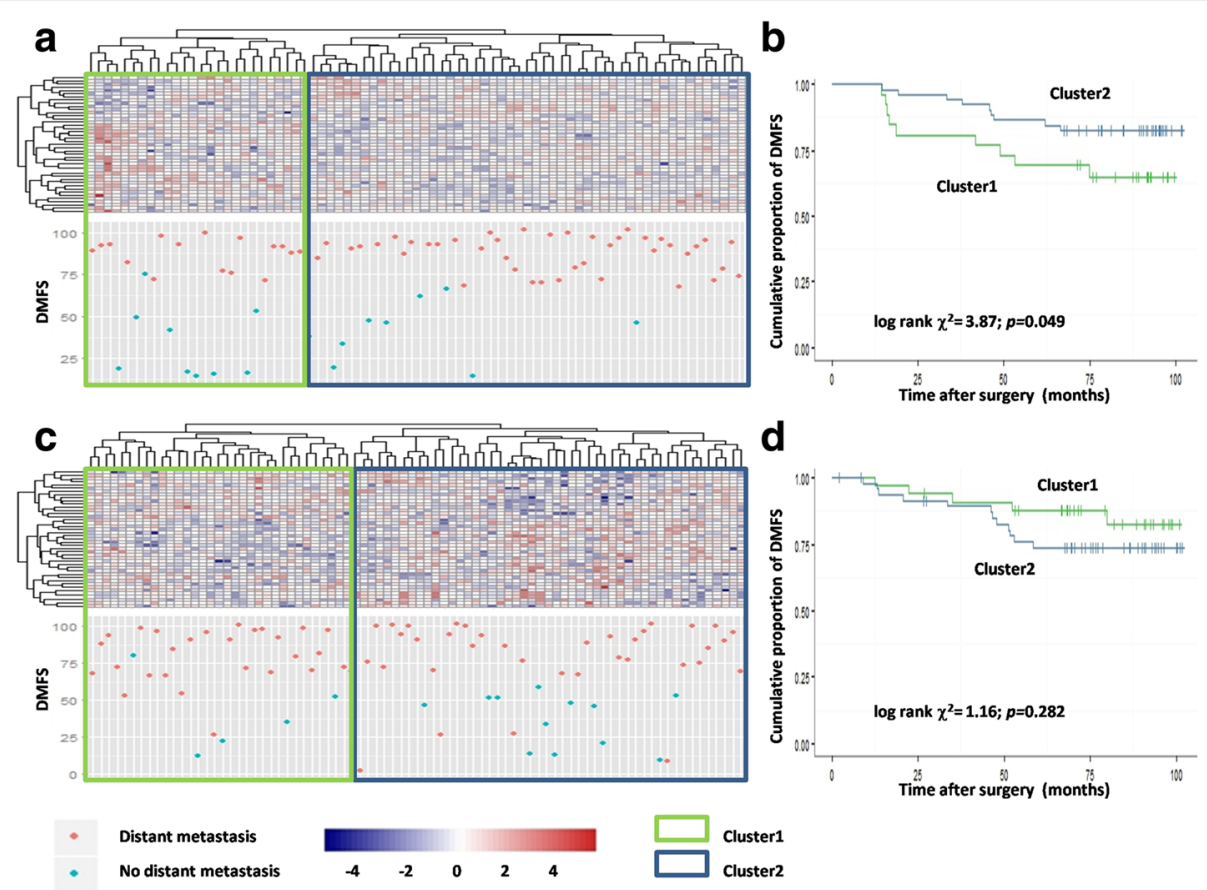

Fig. 4 Relationship between expressions of SNCG correlated genes and radiotherapy stratified survival in validating dataset. Hierarchical clustering result of patients received radiotherapy (a) and patients did not receive radiotherapy $(\mathbf{c})$ in breast cancer dataset E-TABM-158 by SNCG-correlated genes; Kaplan-Meier curve of DMFS in patients received radiotherapy (b) and patients did not receive radiotherapy (d), grouped by clustering result of SNCG-correlated genes, in breast cancer dataset E-TABM-158

In this study, to reveal the association between SNCG expression and the radiotherapeutic response, we performed a retrospective analysis in which 238 of 454 breast cancer patients were selected according to the indications for postoperative radiotherapy. The aim of this case-by-case screening was to reduce the heterogeneity of our study cohort. In both subgroups stratified with radiotherapy reception, no clinicopathologic feature was found to be associated with SNCG expression, indicating that our patients' data from different subgroups were comparable. Meanwhile, none of our selected patients have received neoadjuvant chemotherapy, which we believe can minimize the pre-surgical bias between groups of patients. Overall, patients with positive SNCG expression status showed a significantly worse prognosis than patients with negative SNCG expression, which is consistent with the results of previous reports $[17,18]$.

Detailed analysis has revealed that among patients that have received radiotherapy, the diversity of prognosis between the SNCG positive and the SNCG negative groups was significantly expanded among patients without radiotherapy treatment. The negative impact of SNCG on prognosis were twice higher for radiotherapy treated patients than for those who did not receive radiotherapy (medium diversity of OS: -55.0 vs. -20.7 months; medium diversity of DMFS: -63.8 vs. -31.6 months), indicating SNCG is indeed associated with radiotherapeutic efficacy. A COX regression analyses was performed to rule out possible confounding factors. SNCG expression was identified as an independent predictor of OS in patients that received radiotherapy, but not in those that received no radiotherapy. The same analysis was also conducted only for patients of stage III and SNCG was again found to be associated with radiotherapy efficacy (data not shown). Taken together, we hypothesize that positive SNCG may indicate a lack of benefit from radiotherapy for breast cancer patients.

To test whether SNCG expression was correlated with cell response to radiation, two radiation stress related gene sets (SMIRNOV_RESPONSE_TO_IR_2HR_DN [31] and MAYBURD_RESPONSE_TO_L663536_DN [32]) were simultaneously used for gene set enrichment analysis (GSEA) in online breast cancer datasets E-TABM-158 and GSE1456. In both datasets, SNCG expression is negatively enriched with both gene sets, indicating that SNCG was highly correlated with radiation stress. To further elucidate SNCG's association with radiotherapy, the online dataset E-TABM-158 was used for stratified survival analysis. In the radiotherapy subgroup, a high-SNCG expression was suggested to be related with adverse DMFS despite that the statistic was not significant. SNCG and SNCG-correlated genes were recruited to build a 

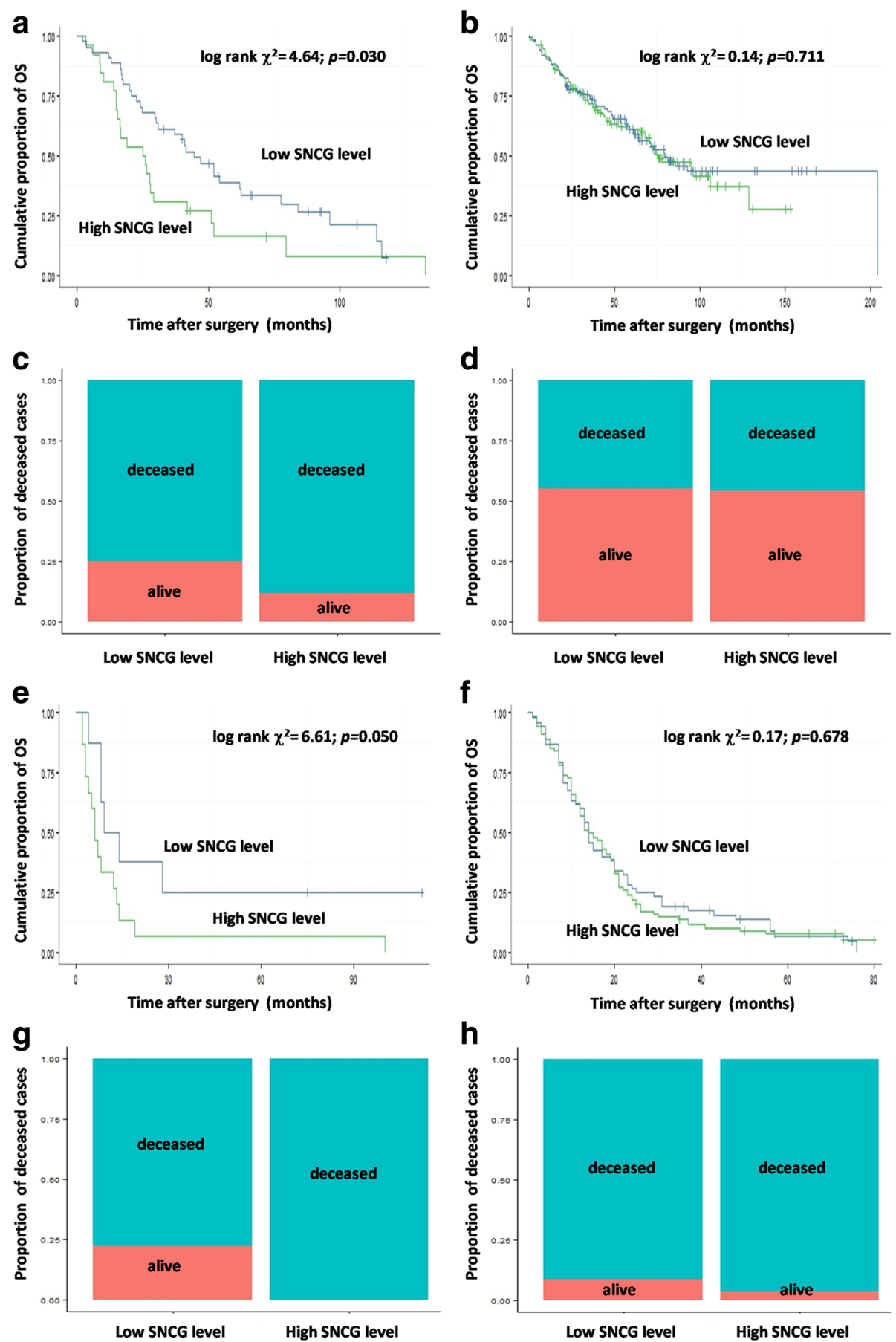

Fig. 5 SNCG expression is associated with radiotherapy related survival in other types of cancer. Kaplan-Meier curve of OS in patients received radiotherapy (a) and patients did not receive radiotherapy (b) in lung cancer dataset CaArray; Mortal rate of patients received radiotherapy (c) and patients did not receive radiotherapy (d) in lung cancer dataset CaArray; Kaplan-Meier curve of OS in patients received radiotherapy (e) and patients did not receive radiotherapy (f) in glioblastoma dataset GSE13041; Mortal rate of patients received radiotherapy (g) and patients did not receive radiotherapy $(\mathbf{h})$ in glioblastoma dataset GSE13041

signature, and signature related prognosis was observed. In contrast, in the non-radiotherapy subgroup, prognosis showed no difference whether grouped by a SNCG or a SNCG-correlated signature. The results presented here strongly support that positive SNCG may serve as a potential biomarker to identify breast cancer patients who are less likely to benefit from radiotherapy. 
How SNCG expression affects sensitivity to radiotherapy still remains unclear. In breast cancer cells, SNCG interacts with phospholipase $C \beta 2$ to modulate $G$ protein activation [34] and it also has the potential to stimulate the ER pathway [35], which may increase cell malignancy. Additionally, SNCG confers resistance to antimicrotubule agents by forming a complex with BubR1 $[11,12,36]$. Considering that the main mechanism of radiotherapy is DNA damage-induced cell cycle arrest and growth inhibition [37], interaction between SNCG and BubR1 may partially explain the role of SNCG in breast cancer cells' response to radiation. Given that radiation could also kill cancer cells effectively through facilitating Reactive Oxygen Species (ROS) generation [38], the possible association between SNCG and ROS-regulated signaling pathway is worth further investigation.

We also explored the possibility that SNCG may serve as a radiotherapy-related biomarker in other types of cancer. The lung cancer dataset CaArray and the glioblastoma dataset GSE13041 were recruited and results similar to breast cancer were achieved, suggesting that SNCG's role in identifying cancer patients less likely to benefit from radiotherapy may be universal in various types of cancer. To make this suggestion stronger, datasets with enough radiotherapy information of more cancer types were needed.

\section{Conclusion}

This is the first study that demonstrates that SNCG could potentially be used as a biomarker to predict a worse patient outcome and less patient benefit as a consequence of radiotherapy for breast cancer. This study also raises an important issue regarding the postoperative adjuvant management of breast cancer. We truly believe that this is an important analysis since patients who have recently undergone a mastectomy and have begun receiving radiotherapy will derive the most benefit from the appropriate risk evaluation and treatment plan selection. Furthermore, an early prediction of radiotherapy treatment efficacy could also potentially improve their quality of life. Considering our results were obtained from a retrospective study, confirmative studies with other cohorts or by prospective, randomly controlled, and double-blinded studies were required.

\section{Additional file}

Additional file 1: Table S1. Prognostic factors of DMFS in univariate analysis of breast cancer patients were or were not treated with radiotherapy. Table S2. Independent predictors of DMFS in multivariate analysis of breast cancer patients were or were not treated with radiotherapy. (DOC $60 \mathrm{~kb}$ )

\section{Acknowledgments}

We deeply appreciate Dr. Lorenzo Finci (Tsinghua University) for the meticulous editing of this manuscript.

\section{Funding}

This study was supported by the National 973 Program of China (2015CB553906, 2013CB910504) and Doctoral Program of Higher Education of China (20110001110050).

\section{Availability of data and materials}

The datasets analyzed during the current study are available in the EBI (http://www.ebi.ac.uk/arrayexpress/experiments/E-TABM-158/) and GEO (http://www.ncbi.nlm.nih.gov/geo/query/acc.cgi?acc=GSE1456) data repository.

\section{Authors' contributions}

LM and CZ carried out the main analysis. LM and CS conceived and designed the study. RM, XL and HY collected the clinical cases and participated in the design of the study. $Y L$ and $R C$ helped to analyze data and revise the manuscript. $C L$ and JG carried out the immunohistology assays and evaluated the IHC grade independently. LM, CZ, LQ and CS draft the manuscript. All authors read and approved the final manuscript.

\section{Authors' Information}

Author list:

\#Li Min: Key Laboratory of Carcinogenesis and Translational Research (Ministry of Education), Department of Biochemistry and Molecular Biology, Peking University Cancer Hospital \& Institute, Beijing 100142, China; Department of Biostatistics and Computational Biology, Dana-Farber Cancer Institute, Harvard School of Public Health, Boston, MA 02115, USA;

minli.mailbox@gmail.com;

\#Cheng Zhang: Key Laboratory of Carcinogenesis and Translational Research (Ministry of Education), Department of Biochemistry and Molecular Biology, Peking University Cancer Hospital \& Institute, Beijing 100142, China; qenya@163.com;

Ruolan Ma: Key Laboratory of Carcinogenesis and Translational Research (Ministry of Education), Department of Thoracic Surgery, Peking University Cancer Hospital \& Institute, Beijing 100142, China; mrllanlan@126.com; Xiaofan Li: Key Laboratory of Carcinogenesis and Translational Research (Ministry of Education), Department of Radiotherapy, Peking University Cancer Hospital \& Institute, Beijing 100142, China; 354275257@qq.com; Hua Yuan: Key Laboratory of Carcinogenesis and Translational Research (Ministry of Education), Breast Center, Peking University Cancer Hospital \& Institute, Beijing 100142, China; 18810530709@126.com;

Yihao Li: Department of Biostatistics and Computational Biology, Dana-Farber Cancer Institute, Harvard School of Public Health, Boston, MA 02115, USA; Department of Biostatistics, UCLA School of Public Health, Los Angeles, CA 90024 USA; yli@jimmy.harvard.edu;

Ruxuan Chen: Peking Union Medical College, Chinese Academy of Medical Sciences, Beijing 100730, China, 15120003091@126.com;

Caiyun Liu: Key Laboratory of Carcinogenesis and Translational Research (Ministry of Education), Department of Biochemistry and Molecular Biology, Peking University Cancer Hospital \& Institute, Beijing 100142, China; liucaiyun@bjcancer.org;

Jianping Guo: Key Laboratory of Carcinogenesis and Translational Research (Ministry of Education), Department of Biochemistry and Molecular Biology, Peking University Cancer Hospital \& Institute, Beijing 100142, China; 542480309@qq.com;

*Like Qu: Key Laboratory of Carcinogenesis and Translational Research (Ministry of Education), Department of Biochemistry and Molecular Biology, Peking University Cancer Hospital \& Institute, Beijing 100142, China; qulike@bjcancer.org;

*Chengchao Shou: Key Laboratory of Carcinogenesis and Translational Research (Ministry of Education), Department of Biochemistry and Molecular Biology, Peking University Cancer Hospital \& Institute, Beijing 100142, China; scc@bjcancer.org.

"First authors: Li Min \& Cheng Zhang contributes equally to this work. *Correspondence authors: Chengchao Shou: Key Laboratory of Carcinogenesis and Translational Research (Ministry of Education), Department of Biochemistry and Molecular Biology, Peking University Cancer Hospital \& Institute, 52 Fucheng Road, Beijing 100142, China, Tel: 0086-1088196766, Fax: 0086-10-88122437, E-mail: scc@bjcancer.org. Like Qu: Department of Biochemistry and Molecular Biology, Peking University Cancer Hospital \& Institute, 52 Fucheng Road, Beijing 100142, China, Tel: 0086-10-88196769, Fax: 0086-10-88122437, E-mail: qulike@bjcancer.org. 


\section{Competing interests}

The authors declare that they have no competing interests.

\section{Consent for publication}

Not applicable.

\section{Ethics approval and consent to participate}

Patients were recruited in the Breast Center at the Peking University Cancer Hospital \& Institute. Treatment and sample collection were approved and supervised by the research ethics committee of Peking University Cancer Hospital \& Institute. Written informed consents were obtained from all participants.

\section{Author details}

${ }^{1}$ Department of Biochemistry and Molecular Biology, Key Laboratory of Carcinogenesis and Translational Research (Ministry of Education), Peking University Cancer Hospital \& Institute, Beijing 100142, China. ${ }^{2}$ Department of Biostatistics and Computational Biology, Dana-Farber Cancer Institute, Harvard School of Public Health, Boston, MA 02115, USA. ${ }^{3}$ Department of Thoracic Surgery, Key Laboratory of Carcinogenesis and Translational Research (Ministry of Education), Peking University Cancer Hospital \& Institute, Beijing 100142, China. ${ }^{4}$ Department of Radiotherapy, Key Laboratory of Carcinogenesis and Translational Research (Ministry of Education), Peking University Cancer Hospital \& Institute, Beijing 100142, China. ${ }^{5}$ Key Laboratory of Carcinogenesis and Translational Research (Ministry of Education), Breast Center, Peking University Cancer Hospital \& Institute, Beijing 100142, China. ${ }^{6}$ Department of Biostatistics, UCLA School of Public Health, Los Angeles, CA 90024, USA. ${ }^{7}$ Peking Union Medical College, Chinese Academy of Medical Sciences, Beijing 100730, China.

\section{Received: 28 August 2015 Accepted: 25 August 2016} Published online: 05 September 2016

\section{References}

1. Torre LA, Bray F, Siegel RL, Ferlay J, Lortet-Tieulent J, Jemal A. Global cancer statistics, 2012. CA Cancer J Clin. 2015;65(2):87-108.

2. Siegel RL, Miller KD, Jemal A. Cancer statistics, 2015. CA Cancer J Clin. 2015; 65(1):5-29.

3. DeSantis C, Ma J, Bryan L, Jemal A. Breast cancer statistics, 2013. CA Cancer J Clin. 2014;64(1):52-62.

4. Holmes MD, Chen WY, Li L, Hertzmark E, Spiegelman D, Hankinson SE. Aspirin Intake and Survival After Breast Cancer. J Clin Oncol. 2010;28(9): 1467-72.

5. Saini KS, Taylor C, Ramirez AJ, Palmieri C, Gunnarsson U, Schmoll HJ, Dolci SM, Ghenne C, Metzger-Filho O, Skrzypski M, et al. Role of the multidisciplinary team in breast cancer management: results from a large international survey involving 39 countries. Ann Oncol. 2011;23(4):853-9.

6. Li Y, Moran MS, Huo Q, Yang Q, Haffty BG. Post-mastectomy radiotherapy for breast cancer patients with t1-t2 and 1-3 positive lymph nodes: a metaanalysis. PLoS One. 2013;8(12):e81765.

7. Luutonen S, Sintonen H, Stormi T, Salminen E. Health-related quality of life during adjuvant radiotherapy in breast cancer. Qual Life Res. 2013.

8. Ji H, Liu YE, Jia T, Wang M, Liu J, Xiao G, Joseph BK, Rosen C, Shi YE. Identification of a breast cancer-specific gene, BCSG1, by direct differential cDNA sequencing. Cancer Res. 1997;57(4):759-64.

9. Lavedan C, Leroy E, Dehejia A, Buchholtz S, Dutra A, Nussbaum RL, Polymeropoulos $\mathrm{MH}$. Identification, localization and characterization of the human gamma-synuclein gene. Hum Genet. 1998;103(1):106-12.

10. Inaba S, Li C, Shi YE, Song DQ, Jiang JD, Liu J. Synuclein gamma inhibits the mitotic checkpoint function and promotes chromosomal instability of breast cancer cells. Breast Cancer Res Treat. 2005;94(1):25-35.

11. Miao S, Wu K, Zhang B, Weng Z, Zhu M, Lu Y, Krishna R, Shi YE. Synuclein Compromises Spindle Assembly Checkpoint and Renders Resistance to Antimicrotubule Drugs. Mol Cancer Ther. 2014;13(3):699-713.

12. Singh VK, Zhou Y, Marsh JA, Uversky VN, Forman-Kay JD, Liu J, Jia Z. Synucleingamma Targeting Peptide Inhibitor that Enhances Sensitivity of Breast Cancer Cells to Antimicrotubule Drugs. Cancer Res. 2007;67(2):626-33.

13. Wan F, Dong L, Zhang F, Wang Y, Chen F, Ni S, Chen Y, Long J. Clinical study of the relationship between -synuclein and the response of neoadjuvant chemotherapy in breast cancer. J Int Med Res. 2013;41(3):743-53.
14. Jia T, Liu YE, Liu J, Shi YE. Stimulation of breast cancer invasion and metastasis by synuclein gamma. Cancer Res. 1999;59(3):742-7.

15. Liang W, Miao S, Zhang B, He S, Shou C, Manivel P, Krishna R, Chen Y, Shi YE. Synuclein y protects Akt and mTOR and renders tumor resistance to Hsp90 disruption. Oncogene. 2014;34(18):2398-405.

16. Chen X, Dunn TN, Akiyama T, Lee HW, Kim JB, Knotts TA, Smith SR, Sears DD, Carstens E, Adams SH. Evaluation of the Synuclein- $\gamma$ (SNCG) Gene as a PPARy Target in Murine Adipocytes, Dorsal Root Ganglia Somatosensory Neurons, and Human Adipose Tissue. PLoS One. 2015;10(3):e0115830.

17. Guo J, Shou C, Meng L, Jiang B, Dong B, Yao L, Xie Y, Zhang J, Chen Y, Budman DR, et al. Neuronal protein synuclein $\gamma$ predicts poor clinical outcome in breast cancer. Int J Cancer. 2007;121(6):1296-305.

18. Wu K, Quan Z, Weng Z, Li F, Zhang Y, Yao X, Chen Y, Budman D, Goldberg $I D$, Shi YE. Expression of neuronal protein synuclein gamma gene as a novel marker for breast cancer prognosis. Breast Cancer Res Treat. 2006;101(3): 259-67.

19. Czekierdowski A, Czekierdowska S, Wielgos M, Smolen A, Kaminski P, Kotarski J. The role of CpG islands hypomethylation and abnormal expression of neuronal protein synuclein-gamma (SNCG) in ovarian cancer. Neuro Endocrinol Lett. 2006;27(3):381-6.

20. Liu C, Dong B, Lu A, Qu L, Xing X, Meng L, Wu J, Eric Shi Y, Shou C. Synuclein gamma predicts poor clinical outcome in colon cancer with normal levels of carcinoembryonic antigen. BMC Cancer. 2010;10:359.

21. He J, Wu J, Xu N, Xie W, Li M, Li J, Jiang Y, Yang BB, Zhang Y. MiR-210 disturbs mitotic progression through regulating a group of mitosis-related genes. Nucleic Acids Res. 2013;41(1):498-508.

22. Hu H, Sun L, Guo C, Liu Q, Zhou Z, Peng L, Pan J, Yu L, Lou J, Yang Z, et al. Tumor Cell-Microenvironment Interaction Models Coupled with Clinical Validation Reveal CCL2 and SNCG as Two Predictors of Colorectal Cancer Hepatic Metastasis. Clin Cancer Res. 2009;15(17):5485-93.

23. Liu C, Qu L, Xing X, Ren T, Zeng Y, Jiang B, Meng L, Wu J, Shou C, Dong B. Combined Phenotype of 4 Markers Improves Prognostic Value of Patients With Colon Cancer. Am J Med Sci. 2012;343(4):295-302.

24. Pawitan Y, Bjöhle J, Amler L, Borg A-L, Egyhazi S, Hall P, Han X, Holmberg L, Huang F, Klaar $S$, et al. Gene expression profiling spares early breast cancer patients from adjuvant therapy: derived and validated in two populationbased cohorts. Breast Cancer Res. 2005;7(6):R953.

25. Chin K, DeVries S, Fridlyand J, Spellman PT, Roydasgupta R, Kuo W-L, Lapuk A, Neve RM, Qian Z, Ryder T, et al. Genomic and transcriptional aberrations linked to breast cancer pathophysiologies. Cancer Cell. 2006; 10(6):529-41.

26. Subramanian A, Tamayo P, Mootha VK, Mukherjee S, Ebert BL, Gillette MA, Paulovich A, Pomeroy SL, Golub TR, Lander ES, et al. Gene set enrichment analysis: A knowledge-based approach for interpreting genome-wide expression profiles. Proc Natl Acad Sci USA. 2005;102(43): $15545-50$.

27. Vamsi M, Cecilia L, Karl-Fredrik E, Aravind S, Smita S. PGC-1a-responsive genes involved in oxidative phosphorylation are coordinately downregulated in human diabetes. Nature Genet. 2005;34(3):6.

28. Lee Y, Scheck AC, Cloughesy TF, Lai A, Dong J, Farooqi HK, Liau LM, Horvath S, Mischel PS, Nelson SF. Gene expression analysis of glioblastomas identifies the major molecular basis for the prognostic benefit of younger age. BMC Med Genomics. 2008;1(1):52.

29. David F, Craig S, Tara M, Vishal N, Casey O, Michael F. The Cancer Biomedical Informatics Grid (CaBIG $\mathrm{G}^{\mathrm{TM}}$ ), Engineering in Medicine and Biology 27th Annual Conference. 2005. p. 4.

30. Chellappan SP, Győrffy B, Surowiak P, Budczies J, Lánczky A. Online Survival Analysis Software to Assess the Prognostic Value of Biomarkers Using Transcriptomic Data in Non-Small-Cell Lung Cancer. PLoS One. 2013;8(12): e82241.

31. Smirnov DA, Brady L, Halasa K, Morley M, Solomon S, Cheung VG. Genetic variation in radiation-induced cell death. Genome Res. 2011;22(2):332-9.

32. Mayburd AL. Ingenuity Network-Assisted Transcription Profiling: Identification of a New Pharmacologic Mechanism for MK886. Clin Cancer Res. 2006;12(6):1820-7.

33. Kang SM, Kim MH, Song KH, Jung SY, Ahn J, Hwang SG, Lee JH, Lim DS, Song JY. Modulation of dendritic cell function by the radiation-mediated secretory protein $\gamma$-synuclein. Cell Death Disc. 2015;1:15011.

34. Golebiewska U, Guo Y, Khalikaprasad N, Zurawsky C, Yerramilli VS, Scarlata S. gamma-Synuclein interacts with phospholipase Cbeta2 to modulate $\mathrm{G}$ protein activation. PLoS One. 2012;7(8):e41067. 
35. Jiang Y, Liu YE, Lu A, Gupta A, Goldberg ID, Liu J, Shi YE. Stimulation of estrogen receptor signaling by gamma synuclein. Cancer Res. 2003;63(14): 3899-903.

36. Manivel P, Muthukumaran J, Kannan M, Krishna R. Insight into residues involved in the structure and function of the breast cancer associated protein human gamma synuclein. J Mol Model. 2010;17(2):251-63.

37. Bai J, Guo XG, Bai XP. Epidermal growth factor receptor-related DNA repair and radiation-resistance regulatory mechanisms: a mini-review. Asian Pac Cancer Prev. 2012;13(10):4879-81.

38. Zhou S, Ye W, Shao Q, Zhang M, Liang J. Nrf2 is a potential therapeutic target in radioresistance in human cancer. Crit Rev Oncol Hematol. 2013; 88(3):706-15.

Submit your next manuscript to BioMed Central and we will help you at every step:

- We accept pre-submission inquiries

- Our selector tool helps you to find the most relevant journal

- We provide round the clock customer support

- Convenient online submission

- Thorough peer review

- Inclusion in PubMed and all major indexing services

- Maximum visibility for your research

Submit your manuscript at www.biomedcentral.com/submit
Biomed Central 